\title{
Análise de canabinóides neutros em derivados de Cannabis via GC/MS
}

\author{
Grijó, D. R. ${ }^{*}$; Cardozo, L. F. ${ }^{1}$ \\ 1 Programa de Pós-Graduação em Engenharia Química, Universidade Estadual de Maringá, Maringá, PR, Brasil. \\ *e-mail: pg49313@uem.br
}

\begin{abstract}
Resumo
Atualmente a medicina tem grande interesse na espécie Cannabis sativa L., especialmente em dois de seus canabinóides neutros (CN): canabidiol (CBD) e $\Delta^{9}$-tetrahidrocanabinol ( $\Delta^{9}$-THC). Em parceria com a Polícia Científica de Curitiba - Brasil, foram feitas análises, via cromatografia gasosa e espectrometria de massas (GC/MS), da composição e o comportamento térmico de $\mathrm{CN}$ em derivados dessa espécie, com solvente polar e apolar. Testou-se também, em sementes, uma metodologia industrial de limpeza para remover resíduos de $\mathrm{CN}$ aderidos por contato com outras partes da planta. A partir de curvas de calibração, utilizando padrões de CBD, $\Delta^{9}-\mathrm{THC}$ e canabinol (CBN), foi possível fazer análises quantitativas da influência térmica e da metodologia de lavagem. Esses e outros canabinóides foram identificados pela razão massa carga $(\mathrm{m} / \mathrm{z})$. Foi comprovado que o aquecimento de amostras aumenta 0 potencial medicinal, mas também transforma o $\Delta^{9}$-THC em CBN e/ou $\Delta^{8}$-tetraidrocanabinol $\left(\Delta^{8}\right.$-THC), e o CBD em canabielsoin (CBE) e/ou $\Delta^{9}-\mathrm{THC}$. O extrato apolar apresentou maior concentração de CN. Demonstrou-se também a diminuição de $\mathrm{CN}$ nas sementes pelo método de lavagem industrial, bem como o rendimento em óleo esperado em referências. Com esse trabalho foi possível demonstrar a importância de conhecimento do comportamento dos $\mathrm{CN}$ em derivados de Cannabis.
\end{abstract}

\begin{abstract}
Currently the medicine has great interest in the species Cannabis sativa L., especially in two of its neutral cannabinoids $(\mathrm{CN})$ : cannabidiol $(\mathrm{CBD})$ and $\Delta^{9}$-tetrahydrocanabinol $\left(\Delta^{9}-\mathrm{THC}\right)$. In partnership with the Scientific Policy of Curitiba - Brazil, analyses had been made, with gas chromatography and mass spectrometry (GC/MS), of the composition and the thermal behavior of $\mathrm{CN}$ in derivatives of this species, with polar and nonpolar solvent. It was also tested in seeds an industrial cleaning method to remove $\mathrm{CN}$ residues adhered by contact with other parts of the plant. From the calibration curves using the standards CBD, $\Delta^{9}-$ THC and canabinol (CBN), it was possible to make quantitative analysis of the thermal influence and the cleaning method. Those and others canabinnoids were identified by mass/charge ratio $(\mathrm{m} / \mathrm{z})$. It was proven that the heating samples increases the medicinal potential, but also transforms $\Delta^{9}$-THC in CBN and/or $\Delta^{8}$-tetraidrocanabinol $\left(\Delta^{8}\right.$-THC), and CBD in canabielsoin (CBE) and/or $\Delta^{9}$-THC. The nonpolar extract has a higher concentration of $\mathrm{CN}$. Cleaning method reduce $\mathrm{CN}$ in seeds, as well oil yield expected results. With this work it was possible to demonstrate the importance of knowledge of the $\mathrm{CN}$ behavior in Cannabis derivatives.
\end{abstract}

Keywords (Palavras chaves): cannabinoids, gas chromatography, mass spectrometry.

\section{Introdução}

Atualmente no Brasil se tem grande interesse em duas substâncias derivadas da espécie Cannabis sativa L., o canabidiol (CBD) e o $\Delta^{9}$-tetrahidrocanabinol $\left(\Delta^{9}-\mathrm{THC}\right)$ [1], ou seja, $(\mathrm{m} / \mathrm{z})_{\mathrm{CBD}}=(231 ; 174 ; 246 ; 121 ; 193) \mathrm{e}$ $(\mathrm{m} / \mathrm{z})_{\triangle 9-\mathrm{THC}}=(299 ; 314 ; 231 ; 271 ; 243 ; 258)^{[2]}$. Essas substâncias fazem parte de um grupo chamado canabinóides, que são terpenos fenólicos ${ }^{[3]}$ produzidos pela espécie ${ }^{[4]}$, sendo mais de 66 já identificados ${ }^{[5]}$. Atualmente as indústrias vêm utilizando o dióxido de carbono em condições supercríticas $\left(\mathrm{CO}_{2}-\mathrm{SC}\right)$, como solvente, para obter extratos medicinais ${ }^{[6,7,8,9]}$ porém, apesar da eficiência [10,11], ainda vêm sendo utilizando solventes orgânicos ${ }^{[12,13]}$ nesta aplicação.

Em materiais vegetais brutos a maior parte destes canabinóides então na forma de ácido canabidiol (CBDA) e ácido $\Delta^{9}$-tetrahidrocanabinol $\left(\Delta^{9}\right.$-THCA) ${ }^{[14]}$. Um estudo demonstrou uma porcentagem de $\Delta^{9}$-THCA aproximadamente 5,5 vezes maior que de $\Delta^{9}-\mathrm{THC}{ }^{[15]}$. Através da descarboxilação os canabinóides ácidos são submetidos a neutros, sendo o aquecimento em estufa uma técnica indicada para isto [16]. Com isso, torna-se necessário analisar a influência da temperatura e do tempo de aquecimento em estufa, 
também porque, o aquecimento transforma o $\Delta^{9}$-THC em canabinol $(\mathrm{CBN})^{[17]},(\mathrm{m} / \mathrm{z})_{\mathrm{CBN}}=(295 ; 238 ; 310)^{[2]}$, e/ou $\Delta^{8}$-tetrahidrocanabinol ${ }^{[17]},(\mathrm{m} / \mathrm{z})_{\triangle 8-\mathrm{THC}}=(231 ; 314$; $258 ; 271 ; 193)^{[2]}$, e o CBD em cannabielsoin (CBE) [18], $(\mathrm{m} / \mathrm{z})_{\mathrm{CBE}}=(205,1 ; 247,2 ; 147,1 ; 330,3)^{[19]}$, ou em $\Delta^{9}{ }_{-}$ THC ${ }^{[20]}$. Uma vertente científica afirmar que o CBN não ocorre naturalmente e que é obtido apenas da degradação do $\Delta^{9}$-THC [21], outra afirma que este canabinóide também é oriundo do ácido canabinol (CBNA) ${ }^{[14]}$. Além disso, uma terceira fonte afirma que o CBNA é uma forma oxidada do $\Delta^{9}$-THCA ${ }^{[22]}$. O CO2-SC possui alta afinidade pelos canabinóides neutros e baixa pelos canabinóides ácidos [10]. A quantificação exata de canabinóides ácidos e neutros pode ser feita, por exemplo, com cromatografia líquida de alta resolução (HPLC) ou cromatografia gasosa com derivatização ${ }^{[21]}$.

Apesar de afirmado que de uma forma geral as sementes do gênero Cannabis não possuem $\Delta^{9}$-THC [21], alguns estudos mostram que devido ao contato com outras partes das plantas as sementes acabam aderindo canabinóides em sua superfície externa. Dados do Século XXI mostram que em análises do óleo das sementes nos Estados Unidos 117 ppm $(\mu \mathrm{L} / \mathrm{mL})$ era $\Delta^{9}$-THC, na Alemanha 214 ppm e na Suíça 3568 ppm [23]. O elevado teor suíço deve-se ao processamento de sementes de plantas com alto teor de canabinóides ${ }^{[24]}$. Neste país todas as variedades do gênero podem ser cultivadas legalmente, embora 0 limite de $\Delta^{9}$-THC nos alimentos seja regulamentado [25]. De modo geral, é possível reduzir o teor de $\Delta^{9}$-THC com pré-processamentos das sementes. Com o avanço dessas técnicas de limpeza e secagem, as sementes e derivados desenvolvidos nos EUA, diminuíram significativamente o teor desta substância [26, 27], por exemplo, para aproximadamente 20 ppm [23].

Uma indústria canadense testou alguns fluidos para remoção de canabinóides das sementes [28]. De uma forma geral, industrialmente a remoção de impurezas de sementes e/ou grãos, de qualquer gênero, se dá com 0 uso de peneiras e ventilação forçada, sendo ainda a secagem um processamento posterior comum [29]. Devido seu potencial em ácidos graxos a eliminação de canabinóides das sementes pode expandir a utilização dessa matéria prima no Brasil para outras áreas industriais [30], como alimentos [31], combustíveis [32], cosméticos [33], polímeros ${ }^{[34]}$ e etc. A quantidade de $\Delta^{9}$-THC por via oral, em seres humanos, segura para não induzir efeitos neurológicos e psicomotores não podem ultrapassar 1 a $2 \mu \mathrm{g}$ de por quilograma corporal ${ }^{[35]}$.

\section{Materiais e Métodos}

As análises experimentais foram feitas na Polícia Científica de Curitiba - Brasil, entre 18 de Julho de 2016 a 19 de Agosto de 2016. Utilizou-se para as análises um cromatógrafo gasoso Agilent 5975C inert XL MSD, com injeção automática, espectrômetro de massas Triple Axis como detector (CG/MS) e coluna capilar da Thermo Fischer Scientific TR-5MS (30m x $0,25 \mathrm{~mm} \times 0,25 \mu \mathrm{m})$. O gás de arraste utilizado foi 0
Hélio (> 99,9992\%, Premier X50S), produzido pela Air Products. O método de injeção foi splitless e o forno se manteve a $200{ }^{\circ} \mathrm{C}$ por 0,5 minuto, depois $40 \stackrel{\circ}{\circ}$. min $^{-}$até

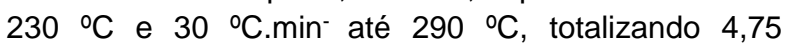
minutos. O eluente utilizado foi metanol $(99,9 \%$, UV/HPLC - Espectroscopico) da Vetec. As áreas foram calculadas por dois métodos de integração diferentes: RTE e ChemStation (CS). Em algumas análise utilizouse n-hexano (95\% UV-IR-HPLC PAI-ACS, da Panreac como solvente, em outras o próprio metanol citado.

\subsection{Curvas de calibração}

Foi utilizado os padrões $\Delta^{9}$-THC, CBD, CBN da Cerilliant Corporation, todos com concentração de $1 \mathrm{mg}$ em $\mathrm{mL}$ de metanol. Foram feitas curvas de calibração para cada padrão utilizando as concentrações 454,5 ; 272,$7 ; 151,5 ; 80,8 ; 26,9 ; 10,4 ; 3,1$ ppm. Para cada curva, Área x ppm, foi analisada a regressão linear e a reta foi ajustada para a origem cartesiana fornecendo a respectiva linearidade $(R)$.

\subsection{Efeito térmico nos canabinóides}

Em todas as amostras padrão descritas anteriormente foi analisado o aquecimento de 180 minutos a $150{ }^{\circ} \mathrm{C}$. Para as amostras de $\Delta^{9}$-THC de 454,5 e 272,7 ppm foram analisados também dois aquecimentos de 30 minutos a $75^{\circ} \mathrm{C}$, sendo que para a segunda concentração ainda analisou-se mais um aquecimento de 25 minutos a $75{ }^{\circ} \mathrm{C}$ posteriormente. Na amostra de CBN com 272,2 ppm também analisou-se um aquecimento sequencial de 40 minutos a $75^{\circ} \mathrm{C}$. Além disso, analisou-se também duas amostras aleatórias de CBN com concentração de 30,74 e 4,50 ppm em dois aquecimentos sequenciais de 60 minutos a $66^{\circ} \mathrm{C}$.

\subsection{Extratos}

\subsubsection{Alta concentração de canabinóides}

Para análise comparativa entre solvente orgânico polar e apolar utilizou-se o metanol e o n-hexano, respectivamente. Para isso, após trituração com aparo manual, $200 \mathrm{mg}$ foi aferido em tubo Falcon e adicionado $20 \mathrm{~mL}$ de solvente, seguida, a temperatura ambiente, de 15 minutos de ultrassom e centrifugação a $3000 \mathrm{rpm}$. Uma quantidade, da mesma amostragem, foi submetida ao aquecimento em estufa a $150 \stackrel{\circ}{\circ} \mathrm{C}$ pequenas porções foram retiradas a cada 15 minutos até completar o tempo de 105 minutos. Depois aferiuse $17 \mathrm{mg}$ em eppendorfs, totalizando 7 para cada tipo de solvente. Em sequência adicionou-se aproximadamente 1,7 mL de solvente, seguido, a temperatura ambiente, de ultrassom por $12 \mathrm{~min}$ e centrifugação a $11000 \mathrm{rpm}$ por $5 \mathrm{~min}$. Em seguida, 200 $\mu \mathrm{L}$ de cada amostra foi transferida para novos eppendorfs. As amostras do solvente apolar foram submetidas a uma corrente de ar para eliminação do 
solvente. Nestas, por fim, foram adicionados 1,2 $\mathrm{mL}$ de metanol, seguido de homogeneização em vórtex e uma nova centrifugação sob mesmas condições anteriores. Nas amostras com solvente polar foram adicionados $1,0 \mathrm{~mL}$ de metanol e feito os procedimentos descritos.

\subsubsection{Sementes}

Com uma peneira e um soprador térmico (Skill 8003) removeu-se parcialmente os canabinóides. Em seguida $2,2 \mathrm{~g}$ foram lavadas com $5 \mathrm{~mL}$ de detergente (Extran ${ }^{\circledR}$ MA 02 Neutro - Merck). Em duplicatas, $1 \mathrm{~g}$ foi aferido em tubo Falcon e adicionado $20 \mathrm{~mL}$ de $\mathrm{n}$-hexano, seguindo de agitação magnética por $60 \mathrm{~min}$ e centrifugação a $3000 \mathrm{rpm}$ por $15 \mathrm{~min}$ a $0 \stackrel{\circ}{\circ}$. O sobrenadante foi extraído para outro Falcon e ao resíduo sólido adicionado mais $10 \mathrm{~mL}$ de $\mathrm{n}$-hexano, seguido de agitação em vórtex por 15 min e centrifugação a $3000 \mathrm{rpm}, 8 \mathrm{~min}$ e $0 \stackrel{\circ}{\circ}$. Juntou-se os sobrenadantes e por fim repetiu-se o procedimento anterior, porém sob agitação em vórtex por $10 \mathrm{~min}$ e centrifugação a $12 \stackrel{\circ}{\circ} \mathrm{C}$. No sobrenadante total foi eliminado 0 solvente sob agitação magnética e aquecimento brando. Após as amostras dos extratos lavados atingirem a temperatura ambiente, aferiu-se a massa para calcular o rendimento. Por fim, adicionouse $4 \mathrm{~mL}$ de metanol e agitação em vórtex. Em sequência $1,8 \mathrm{~mL}$ foi transferido para eppendorfs $e$ estes foram centrifugados a 11000 rpm por $5 \mathrm{~min}$.

\section{Resultados e Discussões}

\subsection{Curvas de calibração}

As relações massa carga obtidas foram $(\mathrm{m} / \mathrm{z})_{\triangle 9-\mathrm{THC}}=$ $(299,1 ; 314,2 ; 231,1 ; 271,1 ; 243,1 ; 258,1),(\mathrm{m} / \mathrm{z}) \mathrm{CBD}=$ $(231,1 ; 246,2 ; 314,2 ; 193,1 ; 174,1 ; 121,1)$ e $(\mathrm{m} / \mathrm{z})_{\mathrm{CBN}}=$ $(295,1 ; 238,1 ; 310,2)$, Os tempos de retenção obtidos estão expressos na Tabela 1 e a linearidade $(R)$ e o coeficiente angular das curvas na Tabela 2.

Tabela 1 - Tempos de retenção obtidos por GC/MS.

\begin{tabular}{|c|c|c|}
\cline { 2 - 3 } \multicolumn{1}{c|}{} & RTE & CS \\
\hline CBD & 4,031 & 4,035 \\
\hline$\Delta 9-\mathrm{THC}$ & 4,368 & 4,371 \\
\hline $\mathrm{CBN}$ & 4,640 & 4,641 \\
\hline
\end{tabular}

Tabela 2 - Linearidades e coeficientes angulares.

\begin{tabular}{|c|c|c|}
\cline { 2 - 3 } \multicolumn{1}{c|}{} & (Linearidade R ; Coeficiente angular) \\
\cline { 2 - 3 } \multicolumn{1}{c|}{} & RTE & CS \\
\hline$\Delta^{9}$-THC & 0,$98 ; 0,01497$ & 0,$98 ; 0,15015$ \\
\hline CBD & 0,$95 ; 0,02258$ & 0,$95 ; 0,22766$ \\
\hline CBN & 0,$97 ; 0,03285$ & 0,$97 ; 0,32342$ \\
\hline
\end{tabular}

\subsection{Efeito térmico nos canabinóides}

\subsection{1 $\Delta^{9}-\mathrm{THC}$}

$\mathrm{Na}$ amostra de 454,5 ppm é possível observar, como mostra a Figura 1, a formação e degradação do CBN.
As linhas pontilhadas demonstram que uma maior quantidade de CBN pode ter sido formada, bem como o $\Delta^{9}$-THC pode ter se esgotado antes.

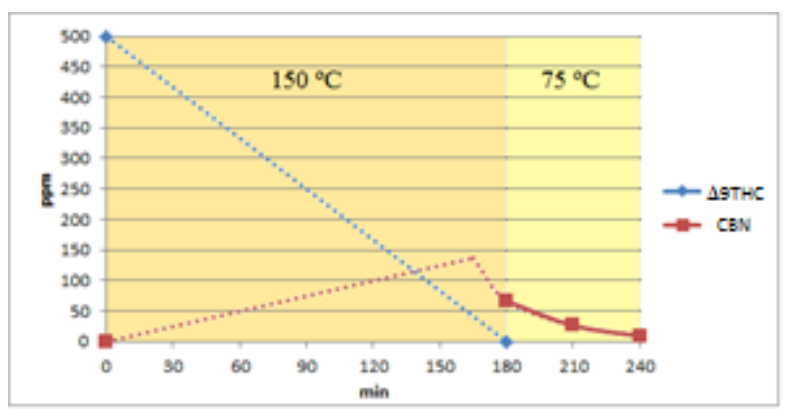

Figura 1 - Efeito térmico em amostra de 454,5 ppm.

Na amostra com 272,7 ppm foi possível observar, além da degradação em CBN, a isomerização em $\mathrm{m} / \mathrm{z}=$ $(231,1 ; 314,2 ; 258,207,0 ; 271,1 ; 193,1)$, possivelmente o $\Delta^{8}$-THC. O pico $\mathrm{m} / \mathrm{z} 207$ é característico da sangria da coluna. Como mostra o Figura 2, nessa análise, foi considerado o máximo de CBN gerado com $180 \mathrm{~min}$, assim como de $\Delta^{8}-\mathrm{THC}$. Como não foi realizada a curva de calibração para $0 \Delta^{8}$-THC, utilizou-se as áreas obtidas no GC/MS. Nas outras concentrações não obteve-se estas substâncias.

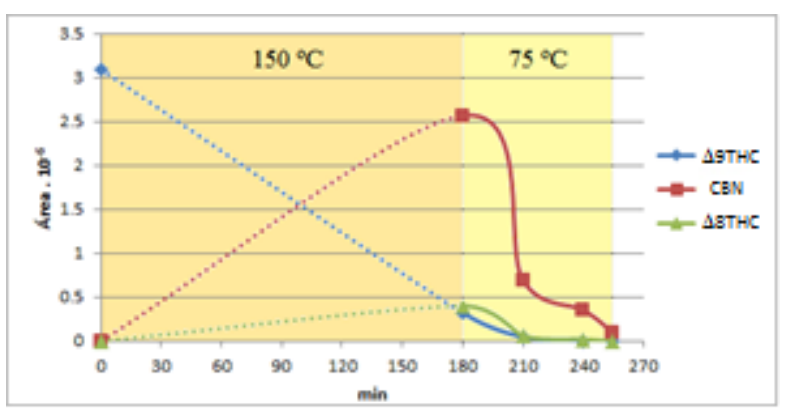

Figura 2 - Efeito térmico em amostra de 272,7 ppm.

Ainda na amostra com 272,7 ppm observou-se a degradação de $0,0075 \mathrm{ppm} \Delta^{9}-\mathrm{THC} .{ }^{\circ} \mathrm{C}^{-} \cdot \mathrm{min}^{-}$, quando este se degradou de 206,55 para 21,47 e depois para $3,20 \mathrm{ppm}$. Nesta amostra também foi observado que a degradação de CBN de 21,01 para 10,77 e depois 2,91 ppm foi com uma variação de 0,0044 ppm. ${ }^{\circ} C^{-} \cdot \min ^{-}$.

\subsubsection{CBN}

Observou-se, pela Tabela 3, que a concentração é diretamente proporcional a degradação térmica.

Tabela 3 - Proporcionalidade na degradação térmica.

\begin{tabular}{|c|c|}
\hline Concentração $(\mathrm{ppm})$ & Variação $\left(\mathrm{ppm} . \mathrm{C}^{-} \cdot \mathrm{min}^{-}\right)$ \\
\hline 272,73 & 0,0056 \\
\hline 30,74 & 0,0028 \\
\hline 4,50 & 0,0004 \\
\hline
\end{tabular}

Nas análises 151,5; 80,9; 26,9; 10,4 e 3,1 ppm observou-se a completa degradação do CBN. 


\subsubsection{CBD}

Em todas as análises observou-se a completa degradação do CBD. Com 454,5 ppm observou-se pelo método de integração CS, no tempo de retenção 4,296, $\mathrm{m} / \mathrm{z}=(207,0 ; 281,0 ; 247,1 ; 147,0 ; 330,2)$ e área $\left(1,549875 \cdot 10^{6}\right)$, possivelmente o CBE. Também um pico com o mesmo tempo característico do $\Delta^{9}$-THC. Na amostra com 272,7 ppm um pico menos pronunciado $\left(0,446972 \cdot 10^{6}\right)$ com aproximadamente o mesmo tempo de retenção, 4,296 min, foi observado.

\subsection{Extratos}

\subsubsection{Alta concentração de canabinóides}

$\mathrm{Na}$ análise comparativa com solvente polar e apolar, sem aquecimento, obteve-se a concentração de $\Delta^{9}$ THC e CBN como mostra a Tabela 4.

Tabela 4 - Composição, em ppm, sem aquecimento.

\begin{tabular}{|c|c|c|}
\hline Hexano & $\Delta^{9}$-THC & CBN \\
\hline RTE & 179,24 & 2,86 \\
\hline CS & 177,70 & 3,05 \\
\hline RTE & 99,75 & 1,73 \\
\hline CS & 99,62 & 1,93 \\
\hline
\end{tabular}

A análise de aquecimento é mostrada no Figura 3, que destaca o hexano em azul e o metanol em vermelho, sendo a parte pontilhada projeções de tendências.

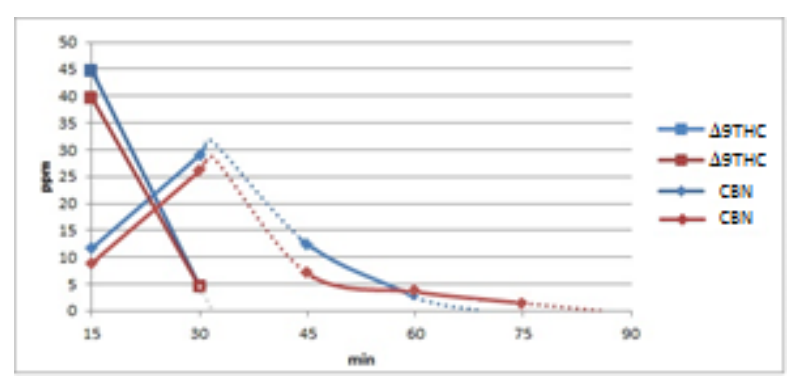

Figura 3 - Comparação de concentração de $\Delta^{9}-\mathrm{THC}$ e CBN em extratos com aquecimento de $150^{\circ} \mathrm{C}$.

Os extratos apresentaram cores gradativas em relação ao tipo de solvente e o tempo de aquecimento. As análises de aquecimento com 75 e $90 \mathrm{~min}$ de hexano e metanol, respectivamente, foram incolores e não apresentaram detecção do CBN.

\subsubsection{Extrato das sementes}

A Tabela 5 apresenta os resultados das sementes.

Tabela 5 - Concentração de $\Delta^{9}$-THC nas sementes.

\begin{tabular}{|c|c|c|}
\cline { 2 - 3 } \multicolumn{1}{c|}{} & \multicolumn{2}{c|}{ Concentração $\Delta^{9}$-THC (ppm) } \\
\cline { 2 - 3 } \multicolumn{1}{c|}{} & Semente & Óleo \\
\hline Sem lavagem & $155,92 \pm 18,48$ & $38.94 \pm 4,66$ \\
\hline Com lavagem & $120,08 \pm 1,12$ & $30.02 \pm 0,28$ \\
\hline
\end{tabular}

O método de lavagem reduziu $23 \%$ de $\Delta^{9}$-THC. A análise sem derivatizante foi aceitável, pois a soma das áreas de canabinóides obtidas na amostra 1 de sementes lavadas, ou seja, $\Delta^{9}-\mathrm{THC}+\mathrm{CBN}$, foi menor que $454,5 \mathrm{ppm}$ de $\Delta^{9}$-THC, que não apresentou degradação do $\Delta^{9}$-THC em outra substância. O rendimento das sementes lavadas se encontra na Tabela 6. O óleo obtido teve coloração amarelada.

Tabela 6 - Rendimento de óleo nas sementes lavadas

\begin{tabular}{|c|c|c|c|}
\cline { 2 - 4 } \multicolumn{1}{c|}{} & Inicial & Final & Teor em óleo (\%) \\
\hline Massa 1 & 0.9857 & 0.3193 & 32.39 \\
\hline Massa 2 & 0.9935 & 0.3005 & 30.25 \\
\hline Média & 0.9896 & 0.3099 & $31.32 \pm 1,07$ \\
\hline
\end{tabular}

\section{Conclusões}

A origem das amostras e o tipo de reagentes são importantes na obtenção de extratos. Comprovou-se a perda de identidade química do $\Delta^{9}$-THC em CBN e $\Delta^{8}$ THC e do CBD em $\Delta^{9}$-THC e CBE. As concentrações observadas de canabinóides neutros, com solvente apolar, com aquecimento a $150 \stackrel{\circ}{ } \mathrm{C}$ até $55 \mathrm{~min}$, foi maior do que com solvente polar.

Os resultados obtidos com extratos das sementes demonstraram boa coerência com a literatura em relação à composição de canabinóides e diminuição destes com a lavagem. Devido à análise de variedades com alto teor de canabinoides e sementes com casca a concentração de canabinóides nas sementes foram relativamente altas.

Mais análises são necessárias, inclusive utilizando HPLC ou GC com derivatização para quantificação exata dos canabinóides ácidos e neutros.

\section{Agradecimentos}

Os autores agradecem a CAPES pelo apoio financeiro ao mestrando e a Polícia Científica de Curitiba pela oportunidade de análise e disponibilidade de material.

\section{Referências}

[1]ANVISA. Agência Nacional de Vigilância Sanitária. Resolução - RDC № 87, de 28 de Junho, 2016.

[2]NIST. National Institute of Standards and Technology. Material Measurement Laboratory. Web book, 2016.

${ }^{[3]}$ MECHOULAM, R., GAONI, Y. Recent advances in the chemistry of hashish. Fortschr, Chem. Org. Naturst. 25, 175-213, 1967.

[4]FASSIO, A. et al. Cáñamo (Cannabis sativa L.). Instituto Nacional de Investigación Agropecuária INIA Uruguay. Noviembre, 2013. 
[5]TURNER, C. E. et al. Constituents of Cannabis sativa L.. J. Nat. Prod. 43, 169-234, 1980.

${ }^{[6] H E M P ~ M E D S . ~ C a l i f o r n i a . ~ U n i t e d ~ S t a t e s . ~} 2016$

[7]BLUE BIRD BOTANICALS. Colorado. United States. 2016

${ }^{[8]}$ ENDOCA. Copenhagen. Denmark. 2016.

[9]BREDOCAN. Veendam. Nertherland. 2016.

${ }^{[10] B R U N E L, ~ H . ~ S u s t a i n a b l e ~ P r o d u c t i o n ~ o f ~ C a n n a b i n o i d s ~}$ with Supercritical Carbon Dioxide Technologies Grade de Master en Génie Chimique, Université de Technologie de Compiègne. 2011.

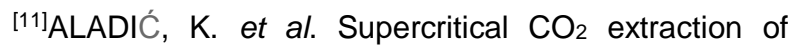
hemp (Cannabis sativa L.) seed oil. Industrial Crops and Products 76 472-478, 2015.

[12]SALAM A. Effect of Cannabis sativa extract on gastric acid secretion, oxidative stress and gastric mucosal integrity in rats. Comparative Clinical Pathology, Vol.24(6), pp.1417-1434, 2015.

[13]KIRALAM, M., et al. Fatty acid composition of hempseed oils from different locations in Turkey. Instituto Nacional de Investigación y Tecnología Agraria y Alimentaria (INIA). Spanish Journal of Agricultural Research 8(2), 385-390, 2010.

[14]STAMBOULI, H.; et al. Cultivation of Cannabs sativa L. in northern Morocco. Bulletin on Narcotics, vol. LVII, Nos, 1 and 2, 2005.

[15]TASCHWER, M.; SCHMID, M. G. Determination of the relative percentage distribution of THCA and $\Delta^{9}$ THC in herbal Cannabis seized in Austria - Impact of different storage temperatures on stability. Forensic Science International 254 167-171, 2015.

[16]VERESS T., et al. Determination of cannabinoid acids by high-performance liquid chromatography of their neutral derivatives formed by thermal decarboxylation in an open re-actor. J. Chromatogr. 520: 339-347, 1990.

[17]GARRET, E. R. Stability of Tetrahydrocannabinols I. College of Pharmacy. University of Florida, 1974.

[18]SHANI, A.; MECHOULAM, R. Cannabielsoic acids. Isolation and synthesis by a novel oxidative cyclization. Tetraedron, v30, issue 15, pages 2437-2446, 1974.

${ }^{[19]}$ RUSSO, E. B.; et al. Phytochemical and genetic analyses of ancient Cannabis from Central Asia. J Exp Botany, Vol. 59, №. 15, pp. 4171-4182, 2008.

${ }^{[20]}$ ADAMS, R. et al. Isomerization of Cannabidiol to Tetrahydrocannabinol, a Physiologically Active Product. Conversion of Cannabidiol to Cannabinol. $\mathrm{J}$ of the American Chem Soc, v62 iss:9 2402 -2405, 1940.
[21]UNODC. United Nations Office on Drugs and Crime. Recommended methods for the identification and analysis of Cannabis and Cannabis products, 2009.

[22]RYMANOWSKI, M. Cannabis - review of the issues related to determination of the total content of $\Delta^{9}-\mathrm{THC}$ and $\Delta^{9}$-THCA-A. Problemy Kryminalistykl 285(3), 2014

${ }^{[23] B R E N N E I S E N ~ R . ~ C h e m i s t r y ~ a n d ~ a n a l y s i s ~ o f ~}$ phytocannabinoids and other Cannabis constituents. Edited by: M. A. EISohly Humana Press Inc, 2007.

[24]LACHENMEIER, D.W. et al. Determination of cannabinoids in hemp food products by use of headspace solid-phase microextraction and gas chromatography-mass spectrometry. Anal Bioanal Chem 378 : 183-189, 2004.

[25]MEDIAVILLA, V. et al. Qualität von Hanfsamenöl aus der Schweiz. Agrarforschung 4, 449-451, 1997.

[26]LESON, G. et al. Evaluating the impact of hemp food consumption on workplace drug tests. J. Anal. Toxicol. 25, 691-698, 2001.

${ }^{[27]}$ ELSOHLY, M. A. Practical challenges to positive drug tests for marijuana. ClinChem49 1037:1038, 2003.

[28]MAASI. Manitoba Association of Agricultural Societies Inc. Development of Hemp Food Products \& Processes, 2000.

[29]LINK, D. J. Fundamentos e estratégias para comercialização de soja e milho e produção de sementes de soja. CCA/UFSC, 2011.

[30]GRIJÓ, D. R., MELO, B. N. "Análise Potencial de Ácidos Graxos Poli-insaturados em Sementes de Cânhamo. Biomassa BR, ed 25, p 30, 2016. Disponível https://issuu.com/revistabiomassabr/docs/revista_bioma ssabr_ed_25_low. Acessado em: 23 Set, 2016

${ }^{[31]}$ Government of Canada. Governments Invest in Bringing Made-in-Manitoba Foods to Market. Disponível <http://news.gov.mb.ca/news/print,index.html?item=334 90\&posted=2014-12-08>. Acesso em: 22 Set, 2016.

[32] LI, S. Y. et al. The feasibility of converting Cannabis oil into biodiesel, BioRTech 101 (21) : 8457-60, 2010.

[33]VOLG, C. R. et al. Hemp as a resource for green cosmetics: yield of seed and fatty acid composition of 20 varieties under the growing conditions of organic farming in Austria. J Ind Hemp 9(1), 51-68, 2004.

[34]SURENDER, R. et al. Synthesis, characterization and degradation behavior of thermoplastic polyurethane from hydroxylated hemp seed oil. Journal of Thermal Analysis and Calorimetry, 2016.

[35]BgVV. German Federal Institute for Consumer Health Protection and Veterinary Medicine. Einsatz von Hanf in Lebensmitteln kann gesundheitlich problematisch sein. BgVV Pressedienst, Berlin, Germany, 1997. 